\title{
Cryogenic Sample Loading into a Magic Angle Spinning Nuclear Magnetic Resonance Spectrometer that Preserves Cellular Viability
}

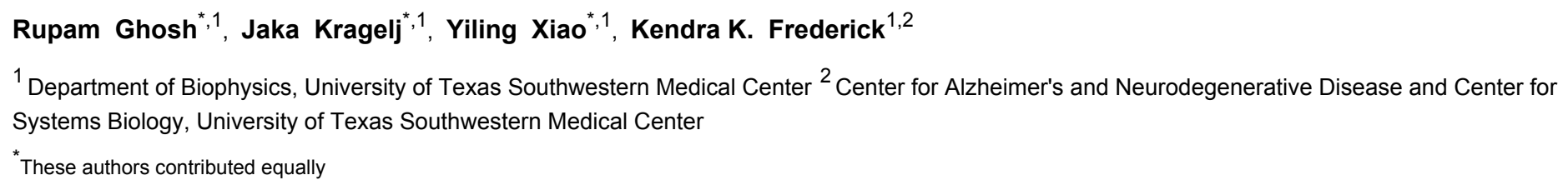

${ }^{1}$ Department of Biophysics, University of Texas Southwestern Medical Center ${ }^{2}$ Center for Alzheimer's and Neurodegenerative Disease and Center for Systems Biology, University of Texas Southwestern Medical Center

*These authors contributed equally

\section{Corresponding Author}

Kendra K. Frederick

kendra.frederick@utsouthwestern.edu

\section{Citation}

Ghosh, R., Kragelj, J., Xiao, Y.,

Frederick, K.K. Cryogenic Sample Loading into a Magic Angle Spinning

Nuclear Magnetic Resonance

Spectrometer that Preserves Cellular

Viability. J. Vis. Exp. (163), e61733,

doi:10.3791/61733 (2020).

\section{Date Published}

September 1, 2020

DOI

$10.3791 / 61733$

\section{URL}

jove.com/video/61733

\section{Abstract}

Dynamic nuclear polarization (DNP) can dramatically increase the sensitivity of magic angle spinning (MAS) nuclear magnetic resonance (NMR) spectroscopy. These sensitivity gains increase as temperatures decrease and are large enough to enable the study of molecules at very low concentrations at the operating temperatures $(\sim 100 \mathrm{~K})$ of most commercial DNP-equipped NMR spectrometers. This leads to the possibility of in-cell structural biology on cryopreserved cells for macromolecules at their endogenous levels in their native environments. However, the freezing rates required for cellular cryopreservation are exceeded during typical sample handling for DNP MAS NMR and this results in loss of cellular integrity and viability. This article describes a detailed protocol for the preparation and cryogenic transfer of a frozen sample of mammalian cells into a MAS NMR spectrometer.

\section{Introduction}

The introduction of dynamic nuclear polarization for magic angle spinning nuclear magnetic resonance spectroscopy can increase the sensitivity of MAS NMR by several orders of magnitude. This has enabled detection of biomolecules at or near their physiological concentrations. DNP can and does provide the sensitivity required to detect an isotopically labeled protein at endogenous $(\sim 1$ $\mu \mathrm{M})$ concentrations in complex biological environment ${ }^{1}$. Because there are well-established protocols to introduce isotopically labeled molecules into unlabeled mammalian cells without affecting their viability, this opens the possibility of studying isotopically enriched biomolecules at their endogenous levels in their native environment. Moreover, because DNP enhancements are more efficient at lower 
temperatures ${ }^{2}, 3,4$, the experimental temperatures for DNP MAS NMR align neatly with those required for longterm storage of viable mammalian cells ${ }^{5}$. However, the conventional method of transferring a sample into a DNP MAS NMR spectrometer subjects it to temperature fluctuation rates that rupture mammalian cells.

MAS NMR experiments require that the sample be rotated about the magic angle at frequencies equal to or greater than the magnitude of the anisotropic interaction for it to be averaged to zero, typically at least $4 \mathrm{kHz}$ and often much higher $^{6,7,8,9}$. Samples are, therefore, packed into rotors that have a finned tip that is used to drive the rotation of the rotor by a stream of gas and have a mark at the other end so the rotation frequency can be monitored by a tachometer. Sample transfer for most MAS NMR instruments is accomplished by injecting the rotor from the exterior of the instrument into the stator at the end of the NMR probe with a stream of dry air or nitrogen gas. After the rotor reaches the stator, which holds the rotor at the magic angle, sample rotation is propelled by an air turbine mechanism. Separate streams of gas support, propel and control the temperature of the rotor. Inserting a rotor into the NMR spectrometer and achieving stable MAS spinning requires finely machined drive tips and tight control of the temperature and pressure of the separate streams of gas. Despite these technical demands, insertion and achieving stable MAS are largely automated for commercial MAS NMR probes for roomtemperature applications.

However, the situation is more complicated for low temperature applications. Samples for low temperature applications are typically inserted into the spectrometer at room temperature and frozen in the stator. In the first minute, the sample temperature decreases quickly $\left(>-100{ }^{\circ} \mathrm{C} / \mathrm{min}\right)$ and the system temperature requires several minutes to equilibrate. Because of the interplay of temperature and pressure, insertion and approaching of the desired MAS are often handled manually for low-temperature applications. Despite the requirement for manual intervention, freezing the rotor inside of the instrument is beneficial because it minimizes the introduction of water and condensation into the probe, which is critical for successful spinning. Not only can condensation and ice build-up from ambient moisture block gas lines, condensation, or frost on the rotor itself can mechanically prevent MAS. Thus, samples for low temperature MAS NMR are typically frozen inside of the instrument at rates that exceed $-100{ }^{\circ} \mathrm{C} / \mathrm{min}$.

Mammalian cells can retain their integrity through a freezethaw cycle if the cooling is $\operatorname{slow}^{5,10,11,12}$, at a rate equal or slower than $1{ }^{\circ} \mathrm{C} / \mathrm{min}$. Alternatively cells also retain their integrity if the cooling rate is ultra-fast ${ }^{13,14,15}$, at a rate faster than $10^{4}{ }^{\circ} \mathrm{C} / \mathrm{min}$. Rates intermediate to these two extremes rupture and kill mammalian cells due to ice crystal formation both inside and outside the cells, even in the presence of cryoprotective agents ${ }^{16}$. The sample cooling rates for a room temperature rotor inside a precooled probe fall between these two extremes, thus to study cryogenically preserved intact viable mammalian cells, samples must be frozen before transfer into the instrument and transferred into the instrument without temperature fluctuations that could damage the sample or accumulation of frost on the rotor that could prevent the rotor from spinning. The protocol describes a method for frost-free, pre-cooled rotor insertion into a cryogenic MAS NMR system for the study of cryogenically preserved intact viable mammalian cell samples. The cryogenic sample transfer described here was developed for NMR characterization of viable intact cells. However, it is applicable to any system where temperature 
fluctuations may compromise sample integrity. This includes any variety of complex systems, such as freeze quenched reactions for chemical and structural characterization of trapped reaction intermediates ${ }^{17,18}$, enzymology ${ }^{19,20}$ or protein folding ${ }^{21,22}$

\section{Protocol}

\section{Culture and cryoprotection of mammalian cells}

1. Culture and harvesting mammalian cells

1. Thaw an aliquot of frozen human embryonic kidney cells (HEK 293).

2. Culture HEK 293 cells in growth media (e.g., DMEM with $10 \%$ fetal bovine serum and $1 \%$ Pen-Strep) at 37 ${ }^{\circ} \mathrm{C}$ with $5 \% \mathrm{CO}_{2}$ in $100 \mathrm{~mm}$ plates for two to three passages (7-10 days).

3. Split the cells and culture in a $150 \mathrm{~mm}$ plate until cells attain $90-95 \%$ confluency.

NOTE: A $150 \mathrm{~mm}$ plate at $>90 \%$ confluency will be sufficient to fill two sapphire rotors with a diameter of $3.2 \mathrm{~mm}$.

4. Harvest cells using $4 \mathrm{~mL}$ of trypsin (see Table of Materials) and $10 \mathrm{~mL}$ of media. Transfer the suspension to a sterile $15 \mathrm{~mL}$ conical and centrifuge at $673 \times g(1000 \mathrm{rpm})$ for $5 \mathrm{~min}$ at room temperature. Remove the supernatant.

5. Wash the cell pellet with phosphate buffered saline (PBS) (pH 7.4, $\left.-\mathrm{CaCl}_{2},-\mathrm{MgCl}_{2}\right)$.

2. Cryoprotection of cells

1. Collect a $50 \mu \mathrm{L}$ cell pellet in a microcentrifuge tube. Prepare a mixture of $50 \mu \mathrm{L}$ of PBS and $18 \mu \mathrm{L}$ of glycerol in a separate tube.
2. Gently mix the $50 \mu \mathrm{L}$ cell pellet with $68 \mu \mathrm{L}$ of glycerolPBS mixture by adding the glycerol-PBS mixture to the top of the pellet and resuspending the pellet by gently tapping the side of the tube until no clumps remain.

NOTE: Gentle pipetting can also be used to resuspend the cell, however, make sure that cellular integrity is not compromised.

\section{Cryopreservation of mammalian cells in an NMR rotor}

1. Transfer of cells into a $3.2 \mathrm{~mm}$ sapphire rotor.

1. To make a funnel, cut a $200 \mu \mathrm{L}$ pipette tip and insert the narrow end of the cut pipette tip into the $3.2 \mathrm{~mm}$ rotor.

2. Transfer the cells into the funnel sitting on the rotor. Place the rotor together with the funnel in a microcentrifuge tube and pellet the cells into the bottom of the rotor by centrifugation at $673 \times \mathrm{g}$ for 2-3 min at room temperature.

3. Remove the supernatant and any excess sample from the rotor. Repeat these two steps until the rotor is fully packed with the cells.

NOTE: Optionally, determine the cellular viability of the sample before freezing. Resuspend $10 \mu \mathrm{L}$ of the excess cell pellet in $100 \mu \mathrm{L}$ of FBS-free DMEM. Mix 10 $\mu \mathrm{L}$ of the suspension with $0.4 \%$ trypan blue solution and immediately assess viability using an automated cell counter. Use only FBS free media to dilute cells as serum interferes with trypan blue staining.

4. Seal the rotor with a silicon plug using commercially available packing tool. 
5. Close the rotor with a ceramic drive tip by pressing it vertically downward. Avoid touching the delicate fins on the side of the drive tip.

6. Mark half of the bottom edge of the sapphire rotor with a silver permanent marker and the other half of the bottom edge of the rotor with a black permanent marker to allow accurate monitoring of the spinning of the rotor inside of the spectrometer.

NOTE: Imperfections in the marking of the rotor will prevent accurate counting of the spinning frequency, resulting in the failure to achieve stable spinning. Because markers will not write on a frozen rotor and rotor warming compromises sample integrity, marking the rotor before freezing is a critical step.

2. Cryopreservation of cells inside a $3.2 \mathrm{~mm}$ sapphire rotor.

1. Place a cushion made by a piece of tissue or paper towel under the lid and at the bottom of the cryogenic vial (see Table of Materials).

NOTE: The tissue paper protects the rotor marking from damage incurred by bumping against the sides of cryogenic vials.

2. Place the $3.2 \mathrm{~mm}$ sapphire rotor into the cryogenic vial padded with the tissue paper with marked end facing the bottom of the cryogenic vial.

3. Slow freeze the rotor by placing the cryogenic vial into the controlled rate $\left(-1^{\circ} \mathrm{C} / \mathrm{min}\right)$ cooling container and place the container in $-80{ }^{\circ} \mathrm{C}$ freezer for a minimum of $3 \mathrm{~h}$.

4. Transfer the cryogenic vial containing the frozen rotor to liquid nitrogen storage.

\section{Cryogenic transfer of a frozen sample into the NMR spectrometer}

1. Transport the frozen sample to the NMR facility.

1. Transfer the cryogenic vial containing the frozen rotor to a small dewar filled with liquid nitrogen for transport to the NMR facility.

2. Transfer of frozen rotor to the liquid nitrogen bath.

1. Fill a dry, thermally insulated wide mouth foam dewar with $500 \mathrm{~mL}-1 \mathrm{~L}$ of liquid nitrogen.

2. Transfer the rotor from the cryogenic vial into the wide mouth foam dewar filled with liquid nitrogen.

1. Take the cryogenic vial from the transfer dewar in the hand and hold it just above the surface of the liquid nitrogen to protect it from the atmosphere.

2. While holding the cryogenic vial with the mouth pointing slightly downwards, unscrew the cap and let the rotor slide into the liquid nitrogen bath.

NOTE: Once the cap is unscrewed, the rotor must fall from the cryogenic vial into the liquid nitrogen bath quickly (under 1 second) to prevent condensation from collecting on the rotor. The evaporation of liquid nitrogen forms a "nitrogen cloud" in the wide mouth foam dewar and prevents condensation on the rotor before it is submerged into the liquid nitrogen. Longer exposure of the rotor to air can lead to condensation of moisture on rotor walls which will re-condense into ice.

3. Cryogenic transfer of rotor to NMR sample catcher.

1. Prechill a $1.5 \mathrm{~mL}$ microcentrifuge tube by submerging it in the liquid nitrogen bath. Do not close the tube. 
NOTE: Inspect the rotor before transfer into the microcentrifuge tube. Using tweezers, hold the rotor just below the surface of the liquid nitrogen and check that the rotor markings are intact, that no ice deposits have formed on its walls and that the drive tip is intact. Ice crystal deposits on the rotor walls appear as white powder. Be careful to always hold the rotor by its body and not by the drive tip. Do not scratch the marking off the rotor with the tweezer-tips.

2. Under the surface of the liquid nitrogen bath, use tweezers to transfer the rotor into the microcentrifuge tube with the drive tip facing the bottom of the microcentrifuge tube and the markings facing the opening of the tube.

NOTE: Always dry tweezers before submerging in liquid nitrogen or touching the rotor.

3. Using tweezers, hold the tube containing the rotor under liquid nitrogen by its neck.

4. With a second pair of tweezers in hand, be prepared to submerge the NMR sample catcher in the liquid nitrogen bath and hold it so that it is inclined at an acute angle with respect to the microcentrifuge tube. NOTE: Minimize the time that the sample catcher is in contact with the liquid nitrogen to avoid freezing the O-ring. If the O-ring freezes, it will be very difficult to insert the catcher into the spectrometer.

4. Cryogenic rotor transfer from the NMR sample catcher to NMR spectrometer

NOTE: This step requires two people, one to operate the cryocabinet and one to transfer the sample from the liquid nitrogen into the probe.

1. Place the cryocabinet in ejection mode by pressing 'EJECT' on the cabinet.
NOTE: The ejection mode purges the dry and cold nitrogen gas flow at high pressure from the probe to the atmosphere in order to prevent the entrance of atmospheric moisture.

2. Transfer the rotor into the NMR sample catcher.

1. Insert the open end of the NMR sample catcher into the microcentrifuge tube while still under the surface of the liquid nitrogen.

2. Lift up both the microcentrifuge tube and NMR sample catcher to allow the rotor to fall into the sample catcher. Shake the NMR sample catcher and microcentrifuge tube in case the rotor is stuck on the rim of the sample catcher.

3. Leave the empty microcentrifuge tube on top of the NMR sample catcher to shield the rotor from air.

3. Remove the other empty NMR sample catcher from the probe and lay it on the floor.

4. Transfer the NMR sample catcher containing the rotor to your free hand, remove the microcentrifuge tube and insert it immediately into the probe.

NOTE: If the O-ring freezes, it will be difficult to tighten the sample catcher. Keep applying force until it slides into place.

5. Signal the person operating the cryocabinet to 'STOP EJECT' and 'INSERT'.

NOTE: The insertion mode guides the rotor from the sample catcher into the probe.

6. Spin up the sample to the desired spinning rate (e.g., $12 \mathrm{kHz}$ ) by adjusting the bearing and driving flow pressure controlled by the cryocabinet. (e.g., Immediately increase the bearing gas to $\sim 200 \mathrm{mBar}$ 
and the drive gas to $10 \mathrm{mBar}$. Once the sample spins, increase the bearing gas to $1000 \mathrm{mBar}$ and drive gas to $200 \mathrm{mBar}$. As spinning stabilizes, increase the bearing to $2400 \mathrm{mBar}$ and then increase drive gas from $200 \mathrm{mBar}$ to $1700 \mathrm{mBar}$ over several minutes. VT cooling gas is constant at $\sim 1070 \mathrm{~L} / \mathrm{h}$.)

NOTE: When lifting the microcentrifuge tube and NMR sample catcher out of the liquid nitrogen bath, make sure that microcentrifuge tube has enough liquid nitrogen inside it to surround the rotor. Minimize the time between transferring the rotor into the NMR sample catcher and inserting the NMR sample catcher into the spectrometer. All the steps in 3.4 should be completed within $30 \mathrm{~s}$.

\section{Cryogenic removal of the sample from the NMR spectrometer}

1. Preparation of the liquid nitrogen bath and the cryogenic vial

1. Pour $500 \mathrm{~mL}-1 \mathrm{~L}$ of liquid nitrogen into the wide mouth foam dewar and place the bath under the spectrometer.

2. Pre-cool the cryogenic vial. Submerge the empty cryogenic vial containing a piece of tissue paper in the liquid nitrogen bath.

2. Cryogenic transfer of rotor from probe to cryogenic vial

1. Reduce spinning rate to $0 \mathrm{kHz}$ by ramping down the driving and bearing gas flow and eject the rotor by switching to the ejection mode.

2. Keep the ejection mode on, remove the sample catcher from the probe, drop rotor directly into the wide mouth foam dewar containing liquid nitrogen.
3. Using pre-chilled tweezers, transfer the rotor into prechilled cryogenic vial under the surface of the liquid nitrogen.

4. Cap the cryogenic vial. Prechill the cryogenic vial cap by dipping it into liquid nitrogen. Remove the cryogenic vial containing the rotor and liquid nitrogen from the bath and cap the tube with prechilled cap. Do not tighten the cap so that vaporizing nitrogen can be safely released.

5. Re-submerge the cryogenic vial in liquid nitrogen. The sample can be transferred to longer term liquid nitrogen storage or unpacked immediately for further analysis.

\section{Unpacking rotor and viability measurements}

1. Unpacking rotor and measuring viability

1. Pre-warm serum free media (DMEM) or PBS to $37^{\circ} \mathrm{C}$.

2. Remove rotor from liquid nitrogen. Remove drive tip and silicon plug.

NOTE: Sapphire is an excellent heat conductor. Avoid touching the rotor with your fingers because heat transfer can cause local freeze-thaw events that compromise cellular viability.

2. Measuring viability

1. Add $20 \mu \mathrm{L}$ of warm media to the frozen cell pellet in the rotor and resuspend cells. Remove suspension with a pipette and mix the suspension with $100 \mu \mathrm{L}$ of media.

2. Remove $10 \mu \mathrm{L}$ of cell suspension and mix with equal volume of $0.4 \%$ trypan blue solution $(\mathrm{v} / \mathrm{v})$. Incubate at room temperature for $30 \mathrm{~s}$ to $1 \mathrm{~min}$.

3. Measure viability using automated cell counter. 


\section{Representative Results}

Cryogenic insertion of pre-frozen samples of mammalian cells into the NMR spectrometer supports viability throughout the NMR experiment. Schematics of cryogenic transfer of a frozen sample into a pre-cooled NMR probe is shown in Figure 1. Cellular viability and intactness can be assessed using a variety of methods. Here we used a standard dye-based measure of membrane integrity, which aligns well with other methods ${ }^{23}$. Intact cells are impermeable to trypan blue while cells with compromised membrane integrity are permeable. The number of trypan blue permeable and impermeable cells can be rapidly assessed using an automated cell counter. Using the protocol described here, the trypan blue permeability of mammalian cells after MAS NMR (i.e., at point 5.2.3) is similar to the trypan blue permeability of mammalian cells before any temperature change (i.e., point 2.1.3). However, if cells are slow frozen, then warmed to room temperature before insertion (i.e. following the protocol to point 3 before warming the rotor to room temperature before inserting into the chilled probe), cellular viability as assessed by trypan blue decreases to less than $10 \%$ of cells (Figure 2). Thus, freezing cells inside the spectrometer results in the loss of cellular membrane integrity while cryogenic insertion of frozen samples of mammalian cells supports cell viability throughout the NMR experiment. 


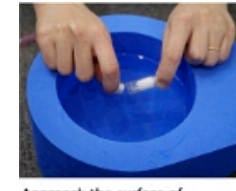

Approach the surface of the liquid nitrogen and unscre
the top of the cryovial.

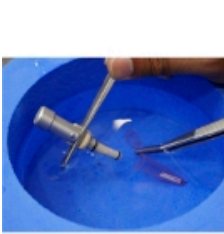

Hold the sample catcher at an angle above the surface recover the rotor from the recover the roto
liquid nitrogen.

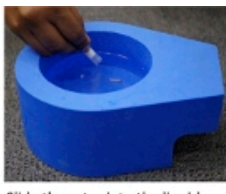

Slide the rotor into the liquid Slide the rotor
nitrogen bath.

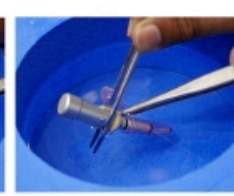

Lift the sample catcher and the tube as soon as the sample catcher is in the microcentrituge tube.

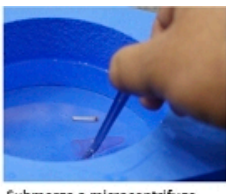

Submerge a microcentrifuge tube using tweezers and hold it in place until it cools dow

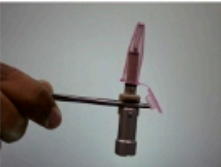

Sometimes the rotor gets caught on the rim of the sample catcher to slip the rotor in.

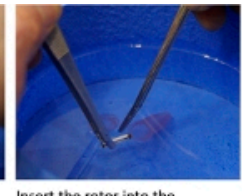

Insert the rotor into the Hold the microcentrifuge tube We recommend pushing the rotor surface and make sure that the In rather than grabbing it with rotor has no frost on it and that

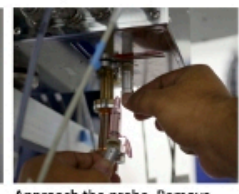

Approach the probe femove the other sample catcher that Lay it on the grount to line. your hand.
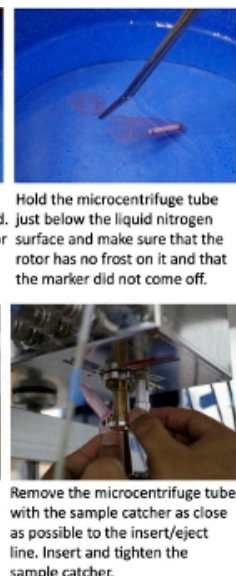

Figure 1: Schematic of cryogenic transfer of a frozen sample into a pre-cooled NMR probe. (A) Approach the surface of the liquid nitrogen and unscrew the top of the cryogenic vial. (B) Slide the rotor into the liquid nitrogen bath. (C) Submerge a microcentrifuge tube using tweezers and hold it in place until it cools completely. (D) Insert the rotor in the microcentrifuge with the drive tip facing the bottom of the tube. Push, rather than grab, with the tweezers. (E) Hold the microcentrifuge tube just below the surface of the liquid nitrogen bath and visually inspect the rotor to ensure that it is frost free and well-marked. (F) Hold the sample catcher at an angle above the surface. (G) Lift the sample catcher and tube out of the liquid nitrogen as soon as the sample catcher is inside the microcentrifuge tube. (H) Shake the sample catcher if the rotor is caught on the rim. (I) Remove the empty sample catcher from the probe and lay it on the ground. (J) Remove the microcentrifuge tube from the sample catcher with the rotor inside, insert, tighten the sample catcher in the probe and press "INSERT" on the control console. Please click here to view a larger version of this figure. 

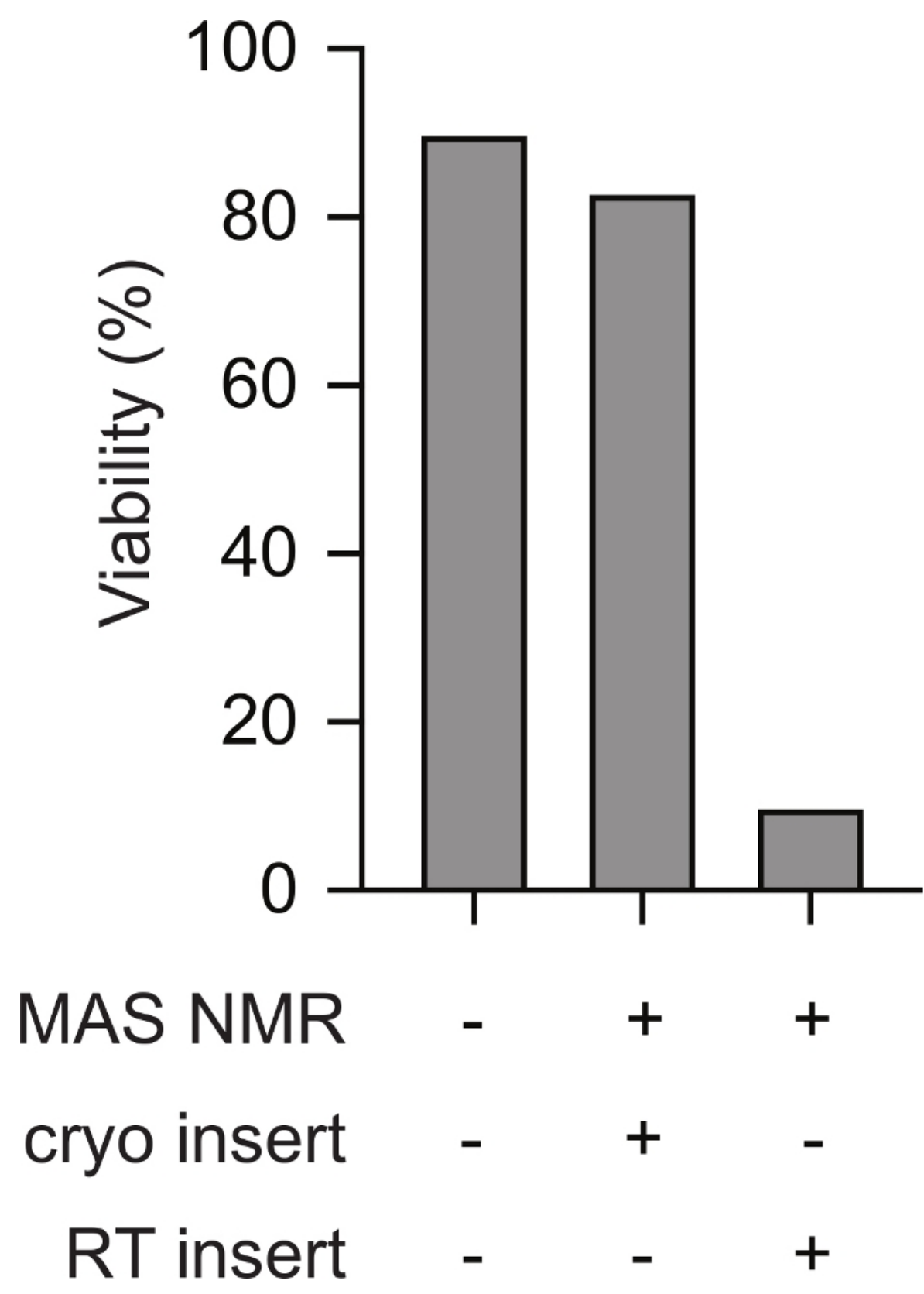

Figure 2: Cryogenic insertion of a pre-frozen sample of mammalian cells results in measurements of cellular intactness that are similar to mammalian cells samples that have never been frozen. The percentage of trypan blue impermeable cells for samples that have never been frozen (e.g. step 2.1.3) is similar to that of cells for samples after MAS NMR (e.g. step 5.2.3 with $12 \mathrm{kHz}$ MAS). Slow frozen cells (e.g. step 3) that were warmed to room temperature before insertion into the NMR instrument that had been pre-cooled to $100 \mathrm{~K}$ had much lower percentages of intact cells after MAS NMR with $12 \mathrm{kHz}$ MAS. Please click here to view a larger version of this figure. 


\section{Discussion}

The cryogenic transfer of frozen samples into an NMR spectrometer is successful in preserving the viability of frozen mammalian cells through the NMR data acquisition. The success of this methodology is demonstrated in pre and post MAS NMR viability measurements. This approach is successful and generalizable to any system where temperature fluctuations may compromise sample integrity. The currently presented protocol is performed with the HEK 293 cell line. Because cryopreservation conditions for many mammalian cell lines are very similar, it is likely that the conditions reported here are translatable to other cellular systems; however, they may require further optimization of cryoprotectants, sample volumes, and freezing rates to attain the same results.

This methodology can be improved upon by unpacking the rotor faster post NMR experiment. This step is currently sub optimal and its execution affects the viability of the cells. Before the cells can be resuspended in media, the drive tip and silicon plug must be removed from the rotor. The sample thaws unevenly when the rotor is held during removal of the drive tip and the silicon plug so shorter rotor handling times result in higher viabilities. The development of rotor holders or other tools to facilitate uniform thawing and quick removal of the drive tip and the silicon plug would aid in making the post-NMR assessment of viability more accurate.

The approach to cryogenic sample loading described in this article is limited to NMR probes that support sample insertion and ejection with the probe in place in the bore of the NMR magnet. While external sample insertion and ejection is standard for commercial DNP systems, custom probes do not always have this option. Also, this approach to cryogenic sample loading may require some modification for NMR probes built to be compatible with different sized rotors. This protocol has been optimized for $3.2 \mathrm{~mm}$ rotors and may require modification if the outer diameter of the sample catcher exceeds the inner diameter of a microcentrifuge tube (e.g., step 3.4.2).

With the application of DNP to MAS NMR, it is now possible to detect proteins and other biomolecules at endogenous physiological concentrations $24,25,26$. This opens the possibility of studying biomolecules within their native environments. Maintenance of cellular integrity and viability throughout the experiment is likely to be critical in connecting the experimental outcomes of the spectroscopy to biological phenomena. Uncontrolled freezing of samples containing purified proteins or cellular lysates does not typically compromise sample quality ${ }^{7}, 27$, although there are some indications that freezing rate may be an important variable even in purified systems ${ }^{28}$. However, samples of mammalian cells need to be frozen at controlled rate if preserving cellular intactness and viability is important for the interpretation. Here we present a protocol for freezing and transferring frozen samples of mammalian cells into a pre-cooled DNP MAS NMR instrument that avoids potentially damaging temperature fluctuations and supports measurement on viable cells.

\section{Disclosures}

The authors have nothing to disclose.

\section{Acknowledgments}

This work was supported by grants from the Cancer Research Prevention \& Research Institute of Texas [RR150076], the National Science Foundation [1751174]; the National Institutes of Health [NS-111236], the Welch Foundation [1-1923-20170325]; the Lupe Murchison Foundation, the Ted 
Nash Long Life Foundation and the Kinship Foundation (Searle Scholars Program) to K.K.F.

\section{References}

1. Costello, W. N., Xiao, Y., Frederick, K. K. DNP-Assisted NMR Investigation of Proteins at Endogenous Levels in Cellular Milieu. Methods Enzymology. 615 (1), 373-406 (2019).

2. Hall, D. A. et al. Polarization-Enhanced NMR Spectroscopy of Biomolecules in Frozen Solution. Science. 276 (5314), 930-932 (1997).

3. Akbey, Ü., Oschkinat, H. Structural biology applications of solid state MAS DNP NMR. Journal of Magnetic Resonance. 269 (1), 213-224 (2016).

4. Matsuki, Y. et al. Helium-cooling and -spinning dynamic nuclear polarization for sensitivity-enhanced solid-state NMR at $14 \mathrm{~T}$ and 30K. Journal of Magnetic Resonance. 225 (1), 1-9 (2012).

5. Miyamoto, Y., Ikeuchi, M., Noguchi, H., Hayashi, S. Longterm Cryopreservation of Human and other Mammalian Cells at $-80{ }^{\circ} \mathrm{C}$ for 8 Years. Cell Medicine. 10 (1), 1-7 (2018).

6. Lopez del Amo, J.M., Schneider, D., Loquet, A., Lange, A., Reif, B. Cryogenic solid state NMR studies of fibrils of the Alzheimer's disease amyloid- $\beta$ peptide: perspectives for DNP. Journal of Biomolecular NMR. 56 (4), 359-363 (2013).

7. Gupta, R. et al. Dynamic nuclear polarization enhanced MAS NMR spectroscopy for structural analysis of HIV-1 protein assemblies. The Journal of Physical Chemistry B. 120 (2), 329-339 (2016).
8. Lim, B. J., Ackermann, B. E., Debelouchina, G. T. Targetable Tetrazine-Based Dynamic Nuclear Polarization Agents for Biological Systems. ChemBioChem. 21 (9), 1315-1319 (2020).

9. Jaudzems, K. et al. Dynamic nuclear polarizationenhanced biomolecular NMR spectroscopy at high magnetic field with fast magic-angle spinning. Angewandte Chemie International Edition. 57 (25), 7458-7462 (2018).

10. Chaytor, J. L. et al. Inhibiting ice recrystallization and optimization of cell viability after cryopreservation. Glycobiology. 22 (1), 123-133 (2012).

11. Mazur, P. Kinetics of water loss from cells at subzero temperatures and the likelihood of intracellular freezing The Journal of General Physiology. 47 (2), 347-369 (1963).

12. Lovelock, J. E., Bishop, M. W. H. Prevention of Freezing Damage to Living Cells by Dimethyl Sulphoxide. Nature. 183 (4672), 1394-1395 (1959).

13. Bald, W. B. The relative merits of various cooling methods. Journal of Microscopy. 140 (1), 17-40 (1985).

14. Akiyama, Y., Shinose, M., Watanabe, H., Yamada, S., Kanda, Y. Cryoprotectant-free cryopreservation of mammalian cells by superflash freezing. Proceedings of the National Academy of Sciences. 116 (16), 7738-7743 (2019).

15. Shi, M. et al. High-throughput non-contact vitrification of cell-laden droplets based on cell printing. Scientific Reports. 5 (1), 17928 (2015).

16. Pegg, D. E. Principles of cryopreservation. Methods in Molecular Biology. 1257, 3-19 (2015). 
17. Ramilo, C. et al. Detection of the covalent intermediate of UDP-N-acetylglucosamine enolpyruvyl transferase by solution-state and time-resolved solid-state NMR spectroscopy. Biochemistry. 33 (50), 15071-15079 (1994).

18. Jeon, J., Thurber, K. R., Ghirlando, R., Yau, W.M., Tycko, R. Application of millisecond time-resolved solid state NMR to the kinetics and mechanism of melittin self-assembly. Proceedings of the National Academy of Sciences. 116 (34), 16717 (2019).

19. Pievo, R. et al. A rapid freeze-quench setup for multifrequency EPR spectroscopy of enzymatic reactions. Chemphyschem. 14 (18), 4094-4101 (2013).

20. Cherepanov, A. V., de Vries, S. Microsecond freezehyperquenching: development of a new ultrafast micromixing and sampling technology and application to enzyme catalysis. Biochimica et Biophysica Acta (BBA) Bioenergetics. 1656 (1), 1-31 (2004).

21. Hu, K.N., Tycko, R. What can solid state NMR contribute to our understanding of protein folding? Biophysical Chemistry. 151 (1), 10-21 (2010).

22. Hu, K.N., Yau, W.M., Tycko, R. Detection of a transient intermediate in a rapid protein folding process by solid-state nuclear magnetic resonance. Journal of the American Chemical Society. 132 (1), 24-25 (2010).

23. Johnson, S., Nguyen, V., Coder, D. Assessment of cell viability. Current Protocols in Cytometry. 64 (1), 9.2.1-9.2.26 (2013).

24. Frederick, K. K. et al. Sensitivity-enhanced NMR reveals alterations in protein structure by cellular milieus. Cell. 163 (3), 620-628 (2015).
25. Van Der Cruijsen, E. A. W. et al. Biomolecular DNPsupported NMR spectroscopy using Site-directed spin labeling. Chemistry. 21 (37), 12971-12977 (2015).

26. Schlagnitweit, J. et al. Observing an antisense drug complex in intact human cells by in-cell NMR spectroscopy. ChemBioChem. 20 (19), 2474-2478 (2019).

27. Debelouchina, G. T. et al. Dynamic nuclear polarizationenhanced solid-state NMR spectroscopy of GNNQQNY nanocrystals and amyloid fibrils. Physical Chemistry Chemical Physics : PCCP. 12 (22), 5911-5919 (2010).

28. Schmidt, T. et al. Submillisecond freezing permits cryoprotectant-free EPR doubled electronelectron resonance spectroscopy. ChemPhysChem. 21, 1224-1229 (2020). 